\title{
Analysis and Design of Prototype Application of Caregivers' Supportive System Phase 1 Alzheimer's Based on a Design Thinking Process
}

\author{
https://doi.org/10.3991/ijoe.v17i13.24979 \\ Chalermpon Kongjit $\left.{ }^{\bowtie}\right)$, Prem Tuntisak \\ Chiang Mai University, Chiang Mai, Thailand \\ chalermpon.k@cmu.ac.th
}

\begin{abstract}
There are 7 million Alzheimer's patients in Thailand, and $10 \%$ of people of 65 years of age or more are in the early stages of this pernicious disease. Alzheimer's reduces the quality of daily life in several ways, as well as having a negative impact on the work performance of the patient's family. Alzheimer's' patients cannot receive proper care at home, despite the good intentions and efforts of family members, but this is the only option available to many families due to the cost of care in an external setting. The tendency for Alzheimer's patients to wander away from home and become lost is even more problematic for their families and this tendency is estimated to worsen as time goes by. Therefore, the purpose of this research is to alleviate the concern of families by preventing their loved ones who are suffering from Alzheimer's from wandering away from home and becoming lost by designing a prototype system that can track and monitor their movements. Design thinking was used in this research as a creative problem-solving approach to produce an application with the core functions of tracking and monitoring the movement of Alzheimer's patients. The application's accuracy was validated by a scenario test, the results of which were discussed based on user feedback.
\end{abstract}

Keywords—-supportive system, phase 1 Alzheimer's, design thinking process

\section{Introduction}

Smartphones equipped with smart technology have several advanced features, including a web-browsing facility, plenty of storage space and a highly accurate monitoring function, which facilitates a rapid response [1][2]. For these reasons, mobile health applications on smartphones have become invaluable tools to improve patients' lives by providing them with essential health information [3-6] based on monitoring their physical condition, tracking their remote location and giving them faster access to health care and consultation. [6-9].

It appears that the number of mobile healthcare applications is constantly increasing, with new applications being launched every day in most of the world's industriallydeveloped countries. According to a recent Statista report, the number of users has increased in line with the number of healthcare applications, which indicates that these 
applications have inevitably become part of everyday life, with consumers downloading 218 billion mobile apps to their connected devices from 2016 to 2020 [10]. For these reasons, many countries in the developed world have launched mobile health applications to assist users to access healthcare services in relation to primary care $(41 \%)$ and prevention (47\%) [11].

Mobile applications designed especially for Alzheimer patients are applied to monitor and track their movements or remind them of their location [12-13]. The benefit of this kind of application is that it can include cognitive and physical therapies, social activities, educational and nutritional programmes, as well as ensuring patients' safety [14-15]. Several smartphone owners currently use them to operate mobile applications that support them in their everyday living with Alzheimer's disease.

It is estimated that there are 7 million Alzheimer patients in Thailand, and $10 \%$ of people aged 65 and over are in the early stages of this pernicious disease [16], which reduces their quality of life due to the progressive decline of memory, thinking, language and the capacity to learn. The estimated utility cost of Thai patients in 2018 , including formal care, informal care, day/night care, daily supplies and medical devices, was estimated to be US\$ 7, 285 per year in rural settings and US\$ 7, 286 per year in urban areas [17]. Since the average monthly household income in Thailand in 2019 was approximately US\$1,212 in Bangkok and approximately US\$ 696 in other regions [18], it is evident that the cost of caring for an Alzheimer's' patient is approximately $50 \%$ of the average income.

The potential risk of caring for an Alzheimer's' patient is the decline of family members' work performance, especially in Thailand, where most family members work outside the home, this means leaving the Alzheimer's patient alone in the house or paying for a daily supply service. Despite these options being wholly inadequate for taking care of people with Alzheimer's, many families have no choice due to the cost of external care facilities [19]. In addition, the stress of caring for Alzheimer's patients is exacerbated by their tendency to wander away from home and become lost or disappear for days due to forgetfulness. As a result, those who care for persons with Alzheimer's disease or dementia tend to suffer from stress, which is detrimental to their physical and mental health. The propensity of Alzheimer's patients to get lost is becoming more problematic for families based on a report in 2020 when it was estimated that 313 people had become lost or missing in 2019 and the trend is increasing year by year [20].

Therefore, based on the several problems carers encounter when caring for someone with Alzheimer's, there is a need to find appropriate tools or smart devices to alleviate the concerns of family members who are obliged to go to work and leave Alzheimer patients alone by preventing the latter from wandering away from the home environment and becoming lost.

Although the use of smart technology to assist carers has increased on a global scale, access to smart systems or services is still problematic in Thailand, despite the existence of many smart devices in the market and the huge cost of taking care of Alzheimer's patients in external settings. Therefore, this research was motivated by the desire to alleviate the concerns of family members who are obliged to care for a relative with Alzheimer's, who has a tendency to leave the home and become lost. Hence, the research questions are based on how to develop an application that can help carers to 
monitor the movement of persons with Alzheimer's or prevent Alzheimer patients from getting lost (disappearing from home) and how to design an appropriate tool or equipment with a low cost.

Therefore, the aim of this research is to analyse and design a prototype system in the form of an application to prevent Alzheimer patients from wandering away from home and getting lost based on the carer's current smart device, and to validate the ability of the prototype to prevent such behaviour in a test scenario before its implementation.

\section{Literature review}

\subsection{Design thinking}

Design thinking is a scientific methodology or creative approach to solving problems [21]. This research applied design thinking as knowledge and innovation management framework to study and clarify requirements and specific knowledge for initiating the development of prototype application". It consists of the following 5 phases based on the modifications of many researchers: [21-25].

- Phase 1: Empathise. This involves understanding and responding to human needs in terms of the problems that need to be solved.

- Phase 2: Define. This involves identifying the core problems.

- Phase 3: Ideate. This involves viewing the problems in alternative ways and identifying innovative solutions.

- Phase 4: Prototype. Having identified the best possible solution for each problem, this phase entails producing a prototype.

- Phase 5: Test. This involves rigorously testing the completed product using the best solutions identified in the prototype phase. A poor design or incomplete prototype can be improved based on the results of this phase by returning to the previous phases.

\subsection{Usability}

Usability is a way to measure whether the defined goal has been effectively and efficiently achieved and ensure that users are satisfied with the product. Usability based on users' suggestions and theoretical criteria was the core consideration in creating the graphical interface in this study [26]. Usability is defined as the performance of the product in terms of ease of access. Based on ISO 9241-11, it is "the extent to which a product can be used by specified users to achieve specified goals with effectiveness, efficiency and satisfaction in a specified context of use." [27].

\subsection{Related work}

Different sensors were used to detect risk when analysing and presenting a viable solution for monitoring Alzheimer patients. Each mobile app was subjected to a variety of tests based on different types of movement in diverse scenarios that could lead it to the failure to detect risk or to misinterpret it, and the results showed that the solution 
mainly achieved the objective. The app was able to monitor the Alzheimer patient, detect accidental falls and give the care-giver relevant information. The only limitation was related to the optimisation of the machine learning algorithm [28].

The mobile application designed in this study was intended to help individuals diagnosed with Alzheimer's disease in the early stages and track their location using Augmented Reality. Based on the development and testing results, the participants were required to perform three tasks due to the heightened efficiency generated through voice interactions on the Augmented Reality interface. No problems were found, despite the evaluated user not having Alzheimer's disease. The application used a verbal alarm as a reminder to assist the location of Alzheimer disease, which was accepted by the evaluated users [29].

\subsection{Stages of alzheimer's}

According to a report from the Alzheimer's Association, patients with Alzheimer's disease pass through five stages, including an early stage, a middle stage and a late stage, as follows: Stage 1: preclinical Alzheimer's disease; Stage 2: mild cognitive impairment due to Alzheimer's disease; Stage 3: mild dementia due to Alzheimer's disease; Stage 4: moderate dementia due to Alzheimer's disease; and Stage 5: severe dementia due to Alzheimer's disease [30]. However, this research is only focused on users in stages 1 to 3, who have difficulty in remembering, experience a lapse in judgment, get lost or misplace items, forget things more often, are more impulsive or exhibit increasingly poor judgment [31].

\subsection{Application's location-based service}

The components and concepts of Location-Based Service (LBS) are associated with mobile devices, communication networks and positioning or location [32]. Location tracking was used in the application prototype in this research to record the current and past location of an object/device based on a GPS service in an android operation system [33]. Several researchers have confirmed that adding GPS tracking to an Android mobile platform is useful and provides an acceptable degree of accuracy [34].

\section{$3 \quad$ Research methodology}

\subsection{Experimental procedures}

A series of design thinking solutions was used to conduct this research and the steps, tool support, target and output of each experimental procedure are illustrated in Table 1. 
Table 1. Experimental procedures

\begin{tabular}{|l|l|l|l|}
\hline $\begin{array}{c}\text { Research } \\
\text { process }\end{array}$ & \multicolumn{1}{|c|}{ Tool support } & \multicolumn{1}{|c|}{ Targets } & \multicolumn{1}{c|}{ Output } \\
\hline Empathise & $\begin{array}{l}\text { Documentary review/ } \\
\text { knowledge extraction or } \\
\text { capturing }\end{array}$ & To clarify root causes & $\begin{array}{l}\text { Problem, factors, require- } \\
\text { ments }\end{array}$ \\
\hline Define & Knowledge Exploration & $\begin{array}{l}\text { To explore and identify the main } \\
\text { problem }\end{array}$ & $\begin{array}{l}\text { Decision making to find a } \\
\text { fixing solution }\end{array}$ \\
\hline Ideate & $\begin{array}{l}\text { Knowledge mapping or } \\
\text { workflow }\end{array}$ & To generate the scope and function & Application work process \\
\hline Prototype & $\begin{array}{l}\text { Blueprint, and software } \\
\text { framework }\end{array}$ & $\begin{array}{l}\text { To present the design application } \\
\text { supporting system }\end{array}$ & $\begin{array}{l}\text { Design framework of ap- } \\
\text { plication supporting sys- } \\
\text { tem }\end{array}$ \\
\hline Test & Scenario test & $\begin{array}{l}\text { To verify the prototype based on the } \\
\text { criteria for assessing the quality of } \\
\text { mHealth apps }\end{array}$ & $\begin{array}{l}\text { Product implementation } \\
\text { and development }\end{array}$ \\
\hline
\end{tabular}

\subsection{Usability test criteria}

The prototype was evaluated by end-users based on a questionnaire (interview), in which the evaluation criteria were categorised by three dimensions, namely, users' requirements, application functionality and graphic user interface. The criteria for the evaluation, which were modified from those of [35-36], are illustrated in Table 2.

Table 2. Evaluation criteria

\begin{tabular}{|l|l|}
\hline \multicolumn{1}{|c|}{ Questions } & Evaluation criteria \\
\hline Application meets the user's requirements (usability) \\
\hline Q1 & 1. Practical use, understand when used \\
\hline Q2 & 2. Simple design \\
\hline Use of application's functions \\
\hline Q3 & 1. Provides accurate and reliable results \\
\hline Q4 & 2. compatibility between core features and user interface \\
\hline Graphic user interface & \\
\hline Q5 & 1. Consistent pattern of menus, icons, colours and fonts \\
\hline Q6 & 2. Appropriate screen size and clearly visible \\
\hline
\end{tabular}

\subsection{Participation}

Five people were selected to participate in individual interviews based on their experience of caring for an Alzheimer's patient at home in order to determine their needs or the features they would like from the new system. It should be noted that the questions they were asked only focused on estimating of the usefulness of the new system and did not include personal information about the patient due to their right to privacy. Therefore, this study was exempted from ethical research requirements due to the type of research. 
Paper-Analysis and Design of Prototype Application of Caregivers' Supportive System Phase 1...

\subsection{Test scenarios}

The performance of the prototype application was tested in terms of its ability to detect the status of a missing patient and alert the carer. This test was divided into three scenarios to recheck and validate the functionality of the system.

1. Short-range scenario: The system was set to remind the carer. This test covered the application's ability to be used at home, in any area of the house. The range covered was approximately 5-15 meters.

2. Medium-range scenario: This scenario tested the ability of the application to be used when walking between rooms or moving around home area. The range covered was approximately 15-25 meters.

3. Long-range scenario: This scenario tested the ability of the application to be used within a village or outside the home environment. The range covered was approximately 45-70 meters.

The smartphone device was used by the patient (role play), while the carer tested the results of the use. The time taken to alert the carer was evaluated compared to the patient's movement. It should be noted that the application was activated by the patient and the researcher applied all the tests in all the scenarios. The application was tested 10 times in each scenario to validate its accuracy. The accuracy of the time consumed between a range of movements and alerting the carer was recorded (when the system sent a notification to the carer).

\subsection{Application framework}

The prototype application framework in this research was developed by Kodular, which is a modern application builder without coding based on the MIT App Inventor [37]. In developing the application, a web browser-based solution was employed to manage the components in terms of customising their appearance and actions. Although this application is not a native app builder, the framework facilitates fast development, and some modules can be downloaded from the library and more easily customised [38].

\section{$4 \quad$ Results}

\subsection{Results of empathise stage}

The empathise stage is focused on acquiring a deeper understanding of the needs of users (carers) and the problems they encounter when attempting to monitor the activities of Alzheimer patients or prevent them from leaving home. The keywords used to classify their existing problems in the Thai language were "Alzheimer patient always get lost away from home, can't be contacted, lose connection, do not answer the telephone, lose contact information, can't be left alone, etc.," and what they wanted from 
a new system were "reminder to carer, alarm when Alzheimer patient leaves home, any tracking function, easy to use, no need of a new device, etc."

In addition, based on the documentary review of the literature, in cases where patients lose their way home [20], the system should be able to prevent their disappearance by extracting or capturing the relevant knowledge of their location and sending it to the carer.

\subsection{Results of define state}

The necessary points for developing a system to monitor the movements of Alzheimer patients or prevent them from leaving home and getting lost were considered in terms of functionality, convenience and accessibility based on the results of the empathies stage. The functions were considered stepwise, as shown in Table 3.

Table 3. Necessary points for developing a system to monitor Alzheimer patients' movements or prevent them from leaving home and getting lost

\begin{tabular}{|l|l|}
\hline \multicolumn{1}{|c|}{ Problems /necessary factors } & \multicolumn{1}{c|}{ Fixing solution } \\
\hline $\begin{array}{l}\text { Difficult to find Alzheimer patients when they } \\
\text { leave home and get lost. } \\
\text { lose connection, do not answer phone) }\end{array}$ & $\begin{array}{l}\text { - Provide a function that alerts carers in cases where Alz- } \\
\text { heimer - patients get lost or cannot get out of the target } \\
\text { area. } \\
\text { - Provide a function that navigates directly to the Alz- } \\
\text { heimer patient's location. }\end{array}$ \\
\hline $\begin{array}{l}\text { Difficult to obtain recall last contact infor- } \\
\text { mation or position of Alzheimer patient }\end{array}$ & - Provide information of spatial data. \\
\hline $\begin{array}{l}\text { A function or system that does not need a new } \\
\text { device. }\end{array}$ & $\begin{array}{l}\text { - A system that operates on carers' current device. } \\
\text { Simple and easy to use. }\end{array}$ \\
\hline
\end{tabular}

\subsection{Results of ideate stage}

The results of this stage were related to the design flow of the system in response to carers' request to provide a function to alert them in cases where the Alzheimer patient is lost or cannot get out of the target area. Figure 1 indicates the workflow of the application function that operates to receive spatial data from the Alzheimer user and send it to the carer for monitoring purposes, while Figure 2 indicates the flowchart of the application work process from the Alzheimer user's side, which explains the logic of the application to operate spatial data with a functional process. Figure 3 and 4 are part of the programme command (core process) to detect and feed data to track users' movements, as indicated in the Kodular blocks. 


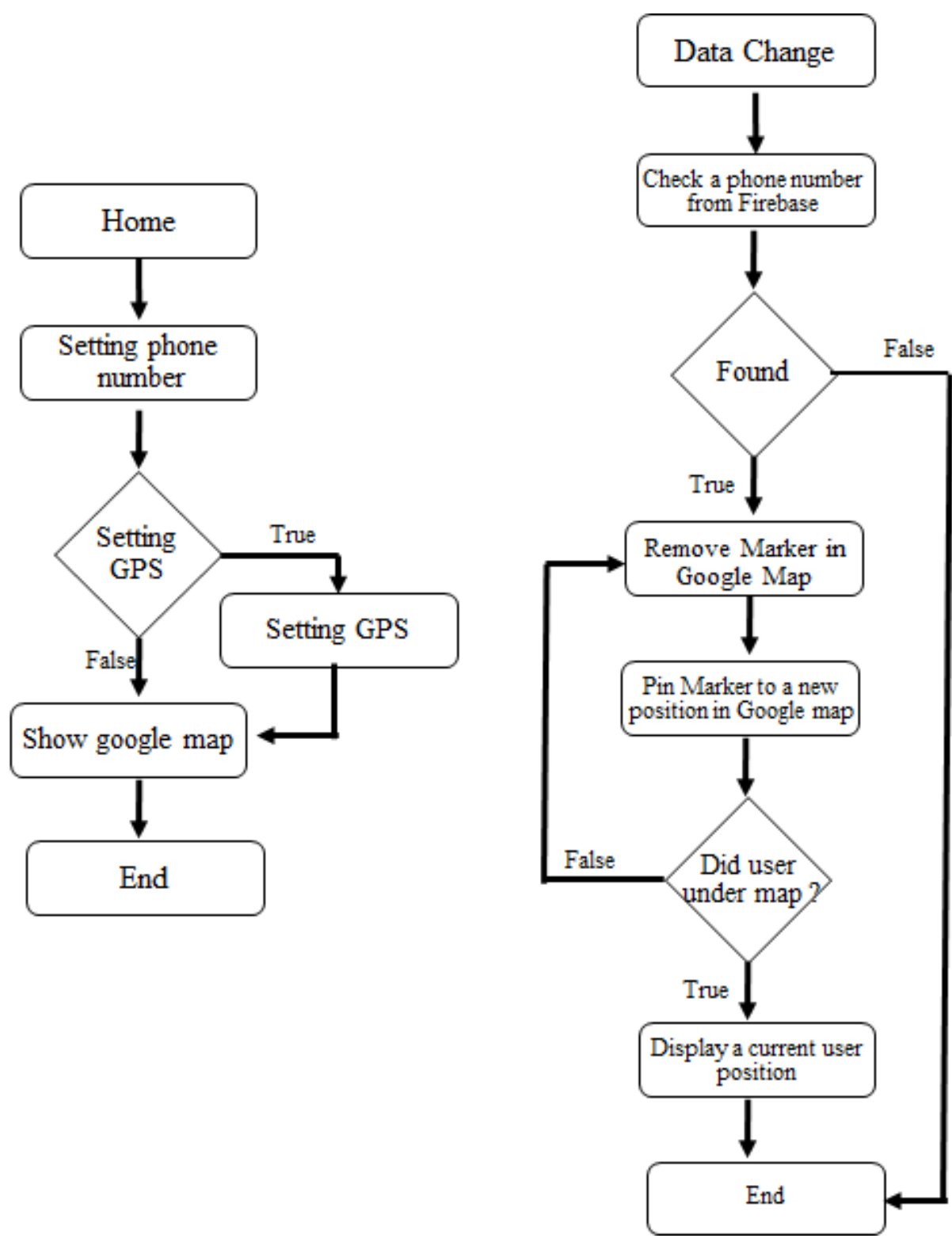

Fig. 1. Flowchart of application work process for carers' side 
Paper-Analysis and Design of Prototype Application of Caregivers' Supportive System Phase 1...

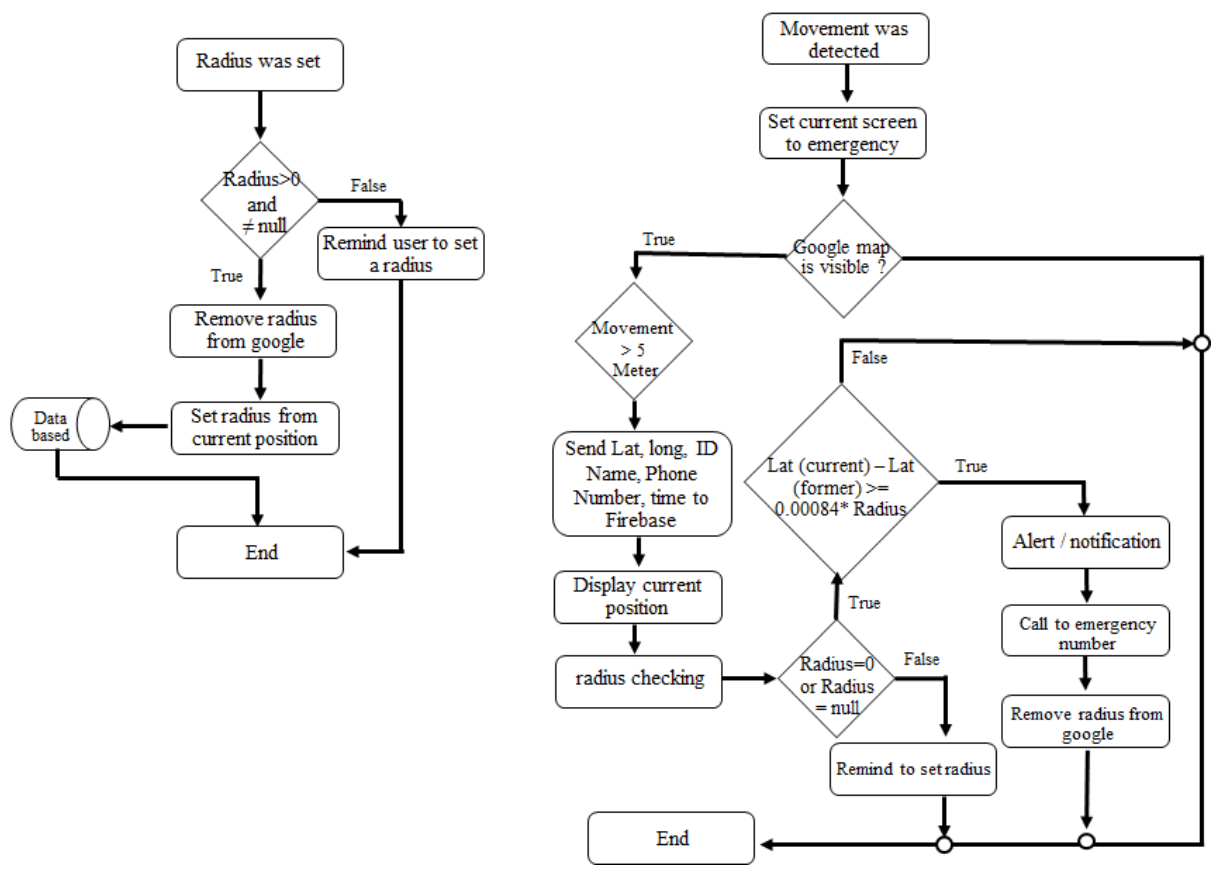

Fig. 2. Flowchart of application work process for Alzheimer patients' side 
Paper-Analysis and Design of Prototype Application of Caregivers' Supportive System Phase 1...

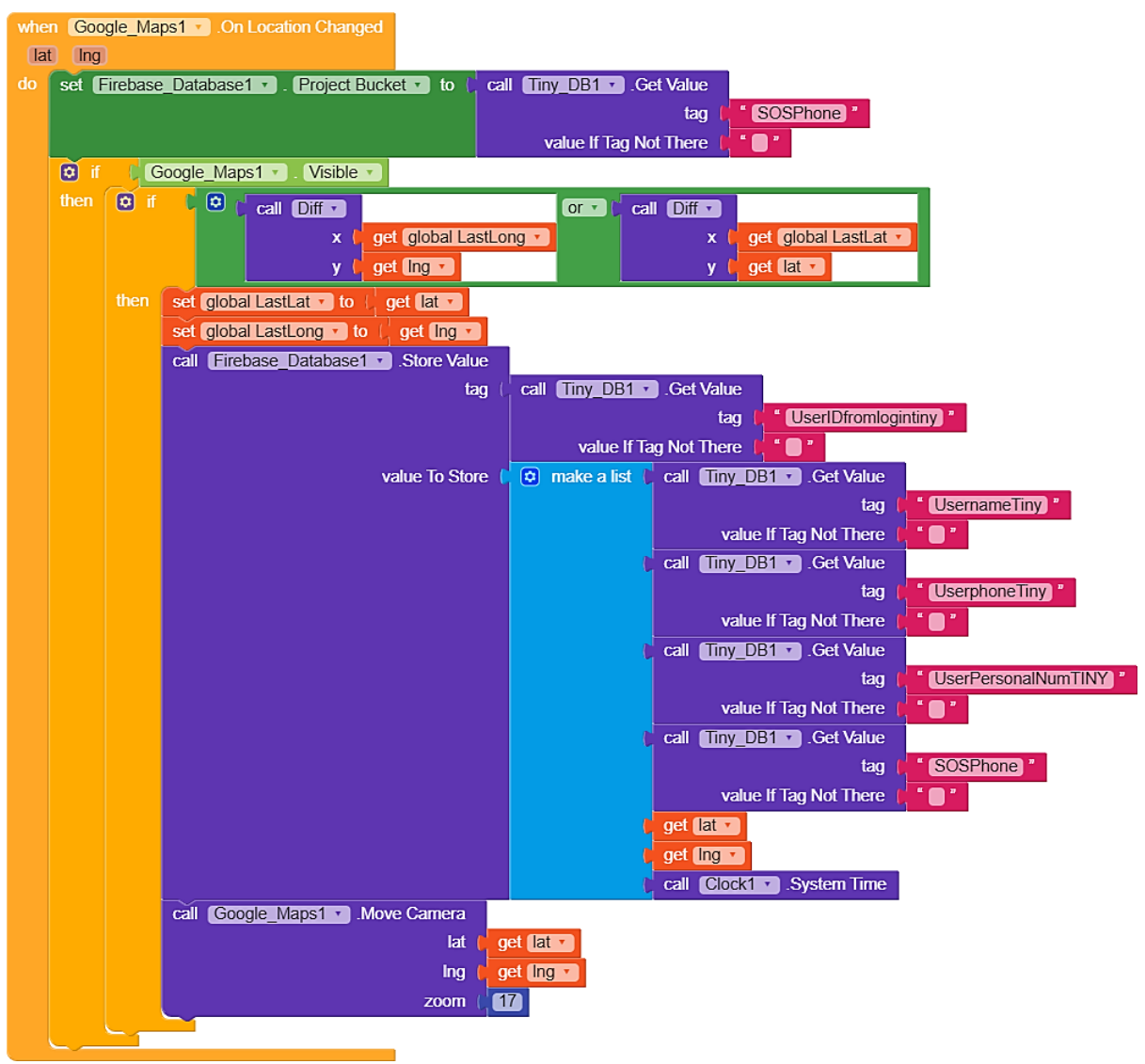

Fig. 3. Programme command (Block command) to detect and feed data to track Alzheimer patients' movements 


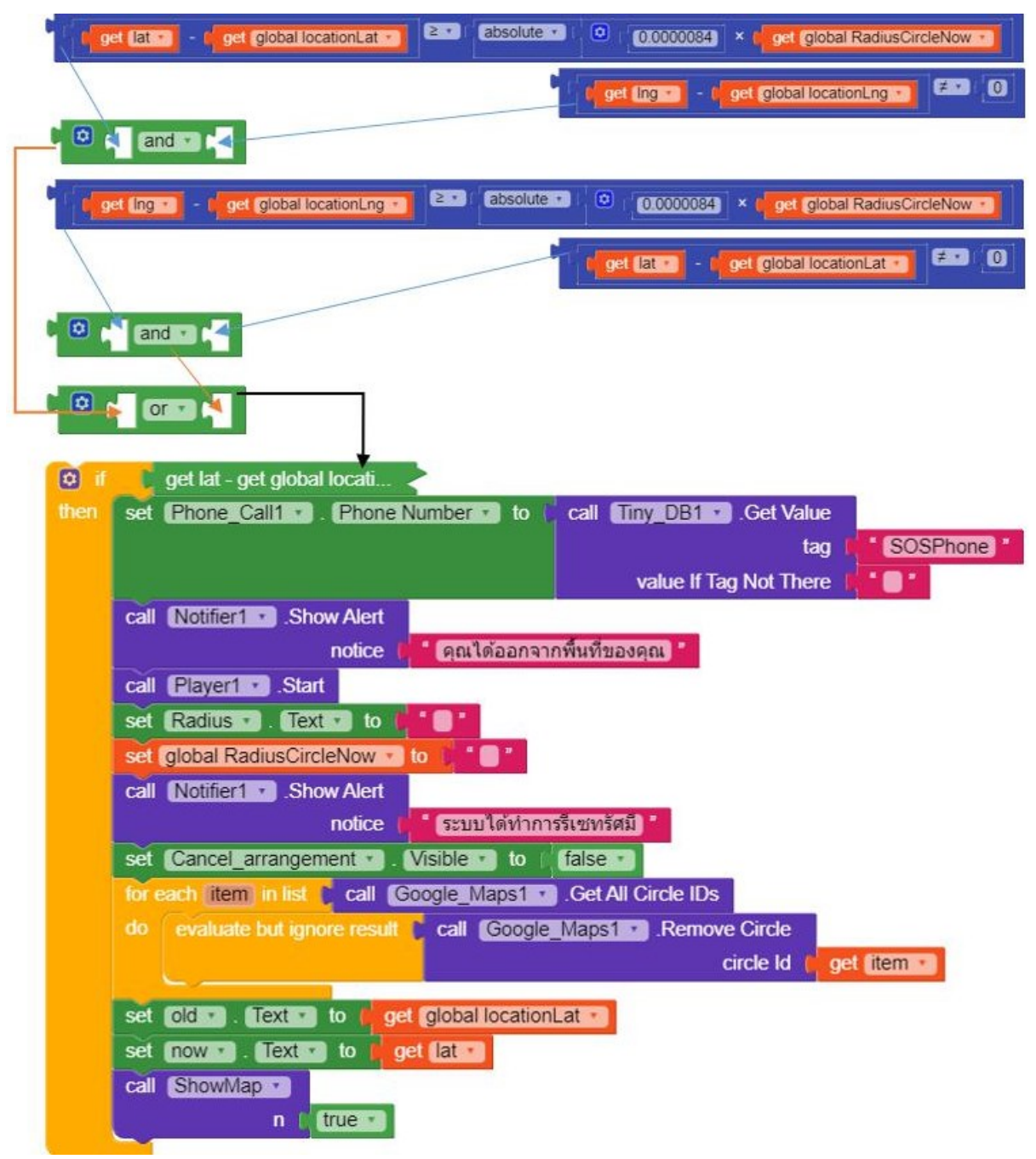

Fig. 4. Programme command (Block command) to detect and feed data to track Alzheimer patients' movements

\subsection{Results of prototype stage}

The results of this stage were related to the blueprint design, which was considered under the flowchart of the work process of designing the application. This design was considered to be appropriate for creating the graphical interface based on the theoretical criteria of ISO 9241-11, which meant that the colours, graphics and layout were based on a simple design that emphasised functions of the application rather than its appearance. An example of screen shot were indicated into Figure 5 and Figure 6. 


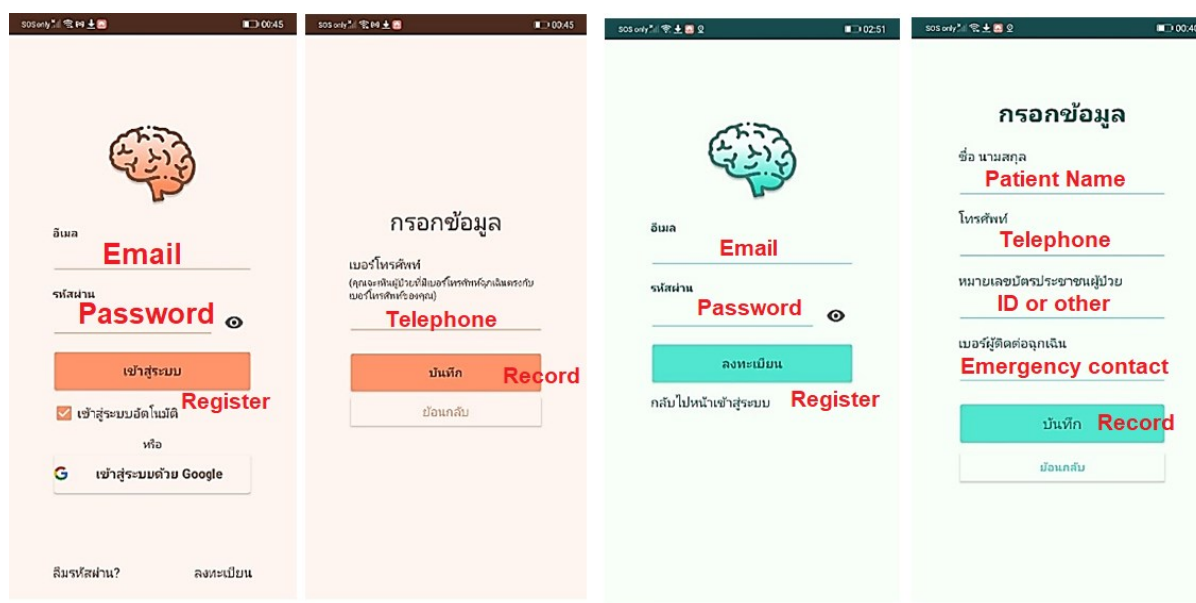

Fig. 5. The two screens on the left-hand side show a screen shot of a function to request the necessary information from the carer, whereas the two on the right-hand side show a screen shot of the function to request information from the users (Alzheimer patients), which was activated by the carer or somebody in the family.
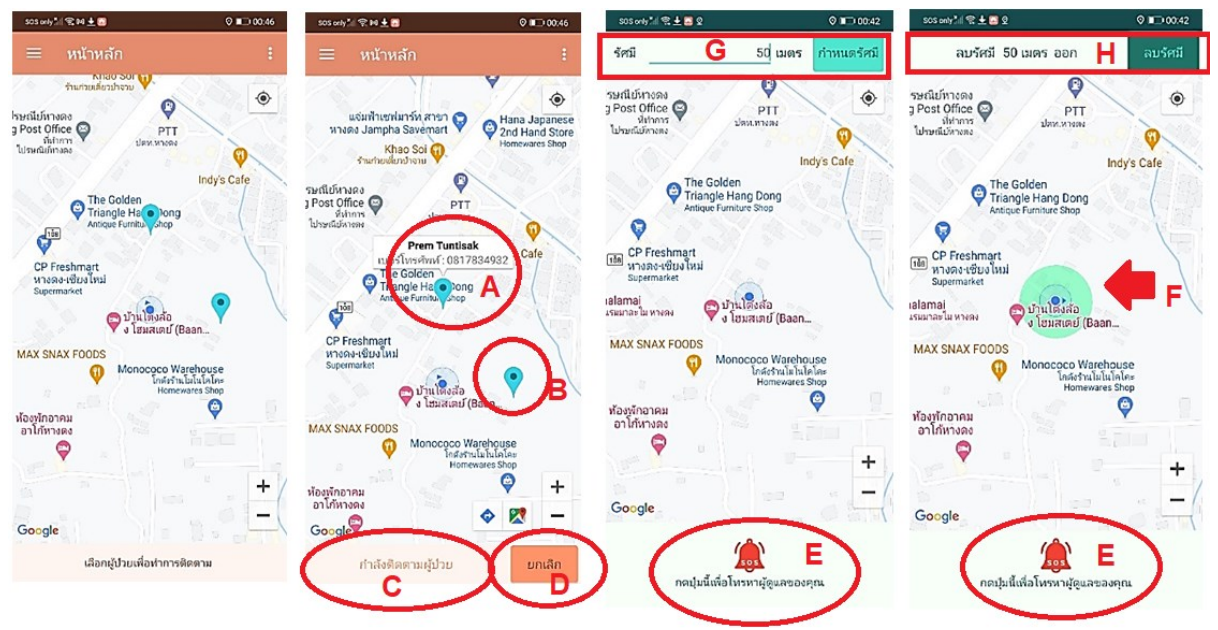

Fig. 6. Core functions of the application are displayed on the sides of both the Alzheimer patient and the carer.

Figure 6 contains the screen shot of application which can be explained as follows: $\mathrm{A}$ and $\mathrm{B}$ are the positions of the user (Alzheimer patient) on the carer's screen.

$\mathrm{C}$ indicates the status of monitoring the user, while $\mathrm{D}$ is the button to cancel the monitoring function.

$\mathrm{E}$ is an icon to call or ask the carer for help. It should be noted that this contains a function to remind the user in Thai to click it when the telephone screen is activated. 
$\mathrm{G}$ is a point to set the boundary of an area by setting a radian for monitoring. This function is activated by the carer or another family member. The boundary of the radian is calculated in meters.

After a boundary has been activated, the user cannot change or fix it until the monitoring mode ends (when $\mathrm{D}$ is clicked). Meanwhile, $\mathrm{H}$ is a point to adjust the boundary or remove it.

$\mathrm{F}$ is the user's current location which is surrounded by the boundary set in point G.

\subsection{Results of the test stage}

At this stage, the prototype application was tested in order to validate its accuracy in each of three scenarios, namely, short-range, medium-range and long-range based on ten tests 1 to 10 , respectively. The results confirmed that the prototype application was more accurate in a long-range scenario than a medium-range and short-range one, respectively. The accuracy of the 10 test counts, which were separated into walk and run to monitor the external movement of the Alzheimer patient, is shown in Table 5, from which it can be seen that it took less time to notify the carer in the walking scenario than it did in the running scenario, with an average time of 8.98 and 16.58 respectively. Moreover, Table 6 contains the results of summarising users' feedback based on the evaluation criteria in Table 2 [35-36].

Table 4. Accuracy Testing

\begin{tabular}{|l|c|c|c|c|c|c|}
\hline \multirow{2}{*}{ Test } & \multicolumn{2}{|c|}{ Shot-range } & \multicolumn{2}{c|}{ Medium-range } & \multicolumn{2}{c|}{ Long-range } \\
\cline { 2 - 7 } & Meter & $\begin{array}{c}\text { Time/ } \\
\text { Second }\end{array}$ & Meter & $\begin{array}{c}\text { Time/ } \\
\text { second }\end{array}$ & Meter & $\begin{array}{c}\text { Time/ } \\
\text { second }\end{array}$ \\
\hline Test 1 & 10.20 & 10.01 & 20.00 & 7.00 & 45.50 & 3.07 \\
\hline Test 2 & 13.50 & 11.50 & 19.50 & 7.88 & 48.15 & 3.75 \\
\hline Test 3 & 7.60 & 9.45 & 21.00 & 7.11 & 50.20 & 3.80 \\
\hline Test 4 & 9.80 & 10.00 & 22.50 & 7.26 & 65.50 & 3.11 \\
\hline Test 5 & 10.80 & 10.19 & 21.00 & 7.12 & 57.12 & 2.19 \\
\hline Test 6 & 9.60 & 9.98 & 19.50 & 7.88 & 50.06 & 3.91 \\
\hline Test 7 & 10.40 & 10.13 & 20.00 & 7.00 & 66.50 & 3.46 \\
\hline Test 8 & 10.30 & 10.08 & 19.40 & 7.87 & 60.45 & 4.50 \\
\hline Test 9 & 8.80 & 9.51 & 18.00 & 7.75 & 63.20 & 4.10 \\
\hline Test 10 & 5.01 & 9.11 & 19.00 & 7.74 & 52.10 & 3.98 \\
\hline Average & 9.60 & 10.00 & 19.99 & 7.46 & 55.88 & 3.59 \\
\hline Sd. & 2.21 & 0.64 & 1.25 & 0.39 & 7.67 & 0.66 \\
\hline
\end{tabular}


Table 5. Accuracy Testing

\begin{tabular}{|l|c|c|}
\hline \multicolumn{1}{|c|}{ Test/count } & Walk (Average $\mathbf{4 . 4} \mathbf{~ k m / h ) ~}$ & Run (Average 8.8 $\mathbf{~ k m} / \mathbf{h})$ \\
\hline Test 1 & 13.4 & 21.04 \\
\hline Test 2 & 7.08 & 25.80 \\
\hline Test 3 & 8.59 & 6.80 \\
\hline Test 4 & 9.62 & 16.3 \\
\hline Test 5 & 9.66 & 23.00 \\
\hline Test6 & 14.35 & 26.25 \\
\hline Test 7 & 13.00 & 6.30 \\
\hline Test 8 & 5.07 & 19.42 \\
\hline Test 9 & 2.84 & 6.34 \\
\hline Test 10 & 6.23 & 14.58 \\
\hline Average & 8.98 & 16.58 \\
\hline Sd. & 3.80 & 7.78 \\
\hline
\end{tabular}

Table 6. Evaluation criteria results

\begin{tabular}{|c|c|c|c|}
\hline Questions & Evaluation criteria & Good & Bad \\
\hline Q1 & $\begin{array}{l}\text { 1. Practical use, under- } \\
\text { stand when used }\end{array}$ & $\begin{array}{l}\text { Some of it is easy to use, fa- } \\
\text { cilitating a fast understand- } \\
\text { ing, simple to use, readable }\end{array}$ & $\begin{array}{l}\text { Small test with just a few words. Some } \\
\text { information the user does not want to } \\
\text { insert in the system }\end{array}$ \\
\hline Q2 & 2. Simple design & Easy to use, high capability & $\begin{array}{l}\text { Some areas of focus are small, but oth- } \\
\text { ers are acceptable. There is a need to } \\
\text { expand the size of the test }\end{array}$ \\
\hline Q3 & $\begin{array}{l}\text { 3. Provides accurate } \\
\text { and reliable results }\end{array}$ & $\begin{array}{l}\text { Finding and monitoring } \\
\text { functions are acceptable } \\
\text { Practical to use }\end{array}$ & $\begin{array}{l}\text { Delay of notification, some issues, ap- } \\
\text { plication restarts itself }\end{array}$ \\
\hline Q4 & $\begin{array}{l}\text { 4. Compatibility be- } \\
\text { tween core features } \\
\text { and user interface }\end{array}$ & $\begin{array}{l}\text { User interface is acceptable, } \\
\text { simple to use, readable }\end{array}$ & Setting area function is a narrow space. \\
\hline Q5 & $\begin{array}{l}\text { 5. Consistent pattern of } \\
\text { menus, icons, colours } \\
\text { and fonts }\end{array}$ & high capability & $\begin{array}{l}\text { Some buttons too small, some text- } \\
\text { boxes restart themselves, some interest- } \\
\text { ing areas are tight }\end{array}$ \\
\hline Q6 & $\begin{array}{l}\text { 6. Appropriate screen } \\
\text { size and clearly visible }\end{array}$ & $\begin{array}{l}\text { Some of it is easy to use, } \\
\text { simple, readable, medium } \\
\text { visibility }\end{array}$ & $\begin{array}{l}\text { Some areas are small, footnote is un- } \\
\text { readable, } \\
\text { Some points of focus on the map too } \\
\text { small }\end{array}$ \\
\hline
\end{tabular}

\section{$5 \quad$ Discussion and conclusion}

The aim of this research was to help to alleviate the concerns of carers or family members who are caring for an Alzheimer patient who is in stages 1 to 3 at home due to the huge cost of external care [19]. In order to achieve this aim, it was necessary to answer two research questions; how to develop an application to help carers to monitor the behaviour of Alzheimer patients and prevent them from wandering away from the 
home environment and becoming lost, and how to design an appropriate tool that can be used on carers' current smartphone and be developed at a low cost.

Design thinking was used in this study as a creative problem-solving method or a solution-based approach to achieve the research aim [21-22].

Each stage of the design thinking process contained certain steps to design an application that had the main function of enabling carers to monitor the movement of Alzheimer patients and notifying them if the patient wandered away from home and was at risk of being lost [20]. In the empathize phrase has supported the extraction of knowledge requirements and provided to define the problem and critical knowledge in define phrase. Moreover, the creativities and innovative solutions enhanced to identify an innovative solution for producing an innovative prototype. The test phrase had done to get the reflection for accuracy.

The application was developed for use by both carers and patients and, according to the results of several scenario tests, it performed long-term monitoring on the carers' current digital devices better than short-term and mid-term monitoring. Therefore, this new prototype application, which was constructed based on design thinking and development, proved to be suitable for monitoring Alzheimer patients outside the home environment due to its ability to notify carers in an accurate and timely manner. Although the monitoring of movement and accuracy of notification was lower inside the house (short-range) than outside (long-range), the application would be very useful if carers were obliged to go to work and leave Alzheimer patients at home alone. If the patients went outside in this scenario, the carers could monitor them and prevent them from getting lost.

In addition, the majority of the comments provided in the feedback based on summarising users' suggestions contained a good mixture of compliments and useful suggestions about issues that should be addressed in the next version of the app. For instance, the android operating system is unstable when accessing geolocation services.

Although the accuracy of the application in notifying carers of the whereabouts of their patient is limited in some environments, it can be a good choice for family members who wish to use smart information technology to assist them in their daily lives and especially to reduce the burden of taking care of Alzheimer patients at home due to the enormous cost of external care in the context of Thai society [17].

Moreover, this research can solve the problems involved in caring for Alzheimer patients at home by designing an appropriate system at low cost, which can be used on an ordinary digital device.

Therefore, the results of this work may enable carers to continue to improve the monitoring of Alzheimer patients' movements in the future to prevent them from getting lost when they leave the home. It is also hoped that the application produced in this project may be a role model to design functions of applications in the future that respond to the needs of Thai society. 


\section{Acknowledgment}

The authors would like to acknowledge the College of Arts, Media and Technology, and Chiang Mai University for the use of its facilities.

\section{$7 \quad$ References}

[1] S. Emine, and S. Marco, "Mobile health access for diabetics in rural Areas of Turkey - results of a survey," Lecture Notes of the Institute for Computer Sciences, Social Informatics and Telecommunications Engineering, Vol. 27, pp.13-20, 2019. https://doi.org/10.1007/978-3642-11745-9 3

[2] A. S. Mohammad Mosa, I. Yoo, and L. Sheet, "A systematic review of healthcare applications for smartphones,”. BMC Medical Informatics and Decision Making, Vol. 12(67), pp. 1-31, 2012. https://doi.org/10.1186/1472-6947-12-67

[3] O. Thinnukool, P. Khuwuthyakorn, P. Wientong, B. Suksat, and N. Waisayanand, "Type 2 Diabetes Mobile Application for Supporting for Clinical Treatment: Case Development Report," International Journal of Online and Biomedical Engineering, Vol. 15(2), pp. 21-38, 2019. https://doi.org/10.3991/ijoe.v15i02.9769

[4] T. Sahebjan, M. Firoozeh, and P. Asiyeh, "Effect of a Mobile Application intervention on Knowledge, Attitude and Practice Related to Healthy Marriage among Youth in Iran," BMC Medical Informatics and Decision Making. Vol. 20:62, 2020. https://doi.org/10. 21203/rs.2.22698/v1

[5] A. Nimmolrat, K. Sutham, and O. Thinnukool, "Patient Triage System for Supporting the Operation Of Dispatch Centres And Rescue Teams," Bmc Medical Informatics And Decision Making, Vol. 21:68, 2021. https://doi.org/10.1186/s12911-021-01440-x

[6] K. Sutham, :P. Khuwuthyakorn, and O. Thinnukool, "Thailand Medical Mobile Application for Patients Triage Base on Criteria Based Dispatch Protocol". BMC Medical Informatics and Decision Making, Vol. 20(1): 66, 2020. https://doi.org/10.1186/s12911-020-1075-6

[7] O. Thinnukool, P. Khuwuthyakorn, and P. Wientong, "Pharmacy Assistant Mobile Application (PAMA): Development and reviews," International Journal of Interactive Mobile Technologies, Vol.11(3), pp. 178-194. 2017. https://doi.org/10.3991/ijim.v11i3.6757

[8] O. Thinnukool, P. Khuwuthyakorn, P. Wientong and T. Panityakul, "Non-prescription medicine mobile healthcare application: Smartphone-based software design and development review," International Journal of Interactive Mobile Technologies, Vol. 11(5), pp.130-146, 2017. https://doi.org/10.3991/ijim.v11i5.7123

[9] A. Nora, K. Mohamed, M. Ashraf, and H. Mowafa, "The prevalence and usage of mobile health applications among mental health patients in Saudi Arabia" Computer Methods and Programs in Biomedicine, Vol.156(2018), pp. 163-168, 2018. https://doi.org/10.1016/ j.cmpb.2017.12.002

Statista Research Department, "Number of mobile app downloads worldwide from 2016 to 2020," Annual number of global mobile app downloads 2016-2020. https://doi.org/10.1787/ $\underline{888934228210}$

[11] NC. Malone, MM. Williams, MC. Smith Fawzi, J. Bennet, C. Hill, JN. Katz, and NE. Oriol, "Mobile health clinics in the United States," International Journal for Equity in Health, Vol.19:40, pp. 1-9, 2020. https://doi.org/10.1186/s12939-020-1135-7

[12] A. Chávez, G. Borrego, J. O. Gutierrez-Garcia, and L.-F. Rodríguez, "Design and evaluation of a mobile application for monitoring patients with Alzheimer's disease: A day center case 
Paper-Analysis and Design of Prototype Application of Caregivers' Supportive System Phase 1...

study," International Journal of Medical Informatics. Vol. 131:103972, (2019), https://doi.org/10.1016/j.ijmedinf.2019.103972

[13] D. M.,Taub, S. B. Leeb, E. C. Lupton, R. T. Hinman, J. Zeisel, and S. Blackler, "The Escort System: A Safety Monitor for People Living with Alzheimer's Disease," IEEE Pervasive Computing, Vol.10 (2), pp. 68-77, 2011. https://doi.org/10.1109/mprv.2010.44

[14] E.D. Jacobson, J. O'Hanlon, and K. Perillo, "The Role of Senior Centers in Mitigating Alzheimer's and Other Forms of Dementia," Institute for Public Administration. Tech, rep. 2019.

[15] L.M. Muriana, and H. Hornung, "Towards Participatory Prototyping with Older Adults Intelligent Assistive Technology for Alzheimer's Disease and Other Dementias: A Systematic Review,” Journal of Alzheimer's Disease, Vol. 56(4), pp.1301-1340, 2017.

[16] Department of Medical Service, “Alzheimer's Disease," Letter of report Department of Medical Service, Ministry of Public Health. Retrieved Jun 2020, from: from: https://pr.moph.go.th/?url=pr/detail/2/02/132212

[17] P. Khongboon, and S. Pongpanich, "Estimating Long-Term Care Costs among Thai Elderly: A Phichit Province Case Study," Journal of Aging Research, Vol. 2018, pp. 1-11, 2018. https://doi.org/10.1155/2018/4180565

[18] Statista Research Department, "Average monthly income per household in Thailand in 2019, by region," Retrieved Jun 2020, from: https://doi.org/10.1787/saeo-2014-graph74-en (2021).

[19] G. Varela, L. Varona, K. Anderson, and J. Sansoni, “Alzheimer's Care At Home: A Focus on Caregivers Strain," Prof Inferm, Vol.64(2), pp. 113-117, 2011.

[20] S. Onmanee, "Thais no plan for Alzheimer's' patients," Silapakorn University. Retrieved Jun 2020, from: https://www.ictsilpakorn.com/ictmedia

[21] Alzheimer's Association, "Basics of Alzheimer's disease," Retrieved Jun 2020, from https://www.alz.org/national/documents/brochure basicsofalz low.pdf

[22] D. Chasanidou, A.A. Gasparini, and E. Lee, "Design Thinking Methods and Tools for Innovation. In: Marcus A. (eds) Design, User Experience, and Usability: Design Discourse," Lecture Notes in Computer Science, Springer, Cham, Vol. 9186. 2015. https://doi.org/ 10.1007/978-3-319-20886-2 2

[23] R. Razzouk, and V. Shute, "What Is Design Thinking and Why Is It Important?," Review of Educational Research September, Vol. 82(3), pp. 330-348, 2012. https://doi.org/10. 3102/0034654312457429

[24] C. Owen, "Design Thinking: Notes on its Nature and Use," Design Research Quarterly, Vol.2(16-27), 2007.

[25] D. Braha, and Y. Reich, "Topological Structures for Modelling Engineering Design Processes," Research in Engineering Design, Vol. 14, pp. 185-199, 2003. https://doi.org/ 10.1007/s00163-003-0035-3

[26] J. Nielsen, "Heuristic evaluation," In: Nielsen J, Mack RL, editors. Usability Inspection Methods. New York: John Wiley \& Sons; 1994. https://doi.org/10.1145/259963.260531

[27] ISO 9241-11, Ergonomic requirements for office work with visual display terminals (VDT's) - Part 11: Guidance on Usabilityll, International Organization for Standardization, Geneva, 1998. https://doi.org/10.3403/01822507u

[28] D. J. L. F. D. V. Rodrigues, "Risk Assessment for Alzheimer Patients, using GPS and Accelerometers with a Machine Learning Approach," Doctoral dissertation, Universidade Nova de Lisboa, 2019. 
Paper-Analysis and Design of Prototype Application of Caregivers' Supportive System Phase 1...

[29] K. M. Kanno, E. A. Lamounier, A. Cardoso, E. J. Lopes, and G. F. M. de Lima, “Augmented reality system for aiding mild Alzheimer patients and caregivers," IEEE Conference on Virtual Reality and 3D User Interfaces (VR). pp. 593-594. 2018. https://doi.org/ $10.1109 / \mathrm{vr} .2018 .8446143$

[30] A. S. Schachter, and K. L. Davis, “Alzheimer's Disease,” Dialogues in Clinical Neuroscience, Vol.2:(2), pp. 91-100, 2000. https://doi.org/10.31887/DCNS.2000.2.2/asschachter

[31] M.V.F. Silva, C.d.M.G. Loures, L.C.V. Alves, and et al., "Alzheimer's Disease: Risk Factors and Potentially Protective Measures,” Journal of Biomedical Science, Vol.26: 33, 2019.

[32] A. Kushwaha, and V. Kushwaha, "Location based Services using Android Mobile Operating System," International Journal of Advances in Engineering and Technology, Vol. 1(1), pp. 14-20, 2011.

[33] R. Rajamoses, and R.B. Sarooraj, Energy-Efficient Location Easy Tracking with Android MobilePhone by Using GPS," Global Journal of Engineering, Design and Technology, Vol.3(2), pp. 1-4, 2014.

[34] R. Pryss, P. Geiger, M. Schickler, J. Schobel, and M. Reichert, "Advanced Algorithms for Location-Based Smart Mobile Augmented Reality Applications," Procedia Computer Science, Vol. 94(2016), pp. 97-104, 2016. https://doi.org/10.1016/j.procs.2016.08.017

[35] J. Lewis, "Psychometric Evaluation of the PSSUQ Using Data from Five Years of Usability Studies," International Journal of Human-Computer Interaction, Vol. 14(3-4), pp. 463-488, 2002. https://doi.org/10.1080/10447318.2002.9669130

[36] R Nouri, R. Niakan Kalhori, Ghazisaeedi M, G. Marchand, and M. Yasini, "Criteria for Assessing the Quality of mhealth Apps :A Systematic Review," Journal of the American Medical Informatics Association, Vol. 25(8), pp.1089-1098, 2018. https://doi.org/10.1093/ja$\underline{\text { mia/ocy } 050}$

[37] W. David, “App Inventor 2: Create Your Own Android Apps,” O'Reilly Media; 2nd edition (November 4, 2014). ISBN-10: 1491906847.

[38] R. S. S. Muhammad, and W. Daru, “The Development of E-Modules Using Kodular Software with Problem-Based Learning Models in Momentum and Impulse Material," Journal of Physics: Conference Series. Vol. 1796: 012078, 2020. https://doi.org/10.1088/1742$\underline{6596 / 1796 / 1 / 012078}$

\section{Authors}

Chalermpon Kongjit, Chalermpon Kongjit, got Ph.D in International and Advanced Japanese Studies: Civil Society from University of Tsukuba in 2017. After he spend many years in Japan to study in Modern Culture and Public Policies from University of Tsukuba in 2005. His current research is focus on Knowledge and Innovation Management, and Collaborative learning. Currently, he works as lecturer in Department of Knowledge and Innovation Management, College of Arts, Media and Technology, Chiang Mai University.

Prem Tuntisak, He is currently student of modern management and information technology. He received his Bachelor of Science in Modern management and information technology from Department of Modern management and information technology, College of Arts, Media and Technology, Chiang Mai University, Chiang Mai, Thailand in 2021.

Article submitted 2021-06-21. Resubmitted 2021-08-02. Final acceptance 2021-08-03. Final version published as submitted by the authors. 